\title{
Einstein Equations in Vacuum as Integrability Conditions
}

\author{
F. J. Chinea \\ Departamento de Métodos Matemáticos de la Física, Facultad de Ciencias Físicas, \\ Universidad de Madrid, Madrid-3, Spain \\ (Received 21 November 1983)
}

\begin{abstract}
The Einstein equations describing gravitational fields in vacuum are written as a compact exterior system of spinor-valued forms. A second system of equations is given, such that their integrability conditions are satisfied by virtue of the Einstein equations. This suggests the possibility of integrating the field equations by means of an inversetype procedure.
\end{abstract}

PACS numbers: $04.20 . \mathrm{Cv}, 04.20 . \mathrm{Jb}$

The solution of the Einstein equations for gravitational fields in vacuum $\left(R_{\mu \nu}=0\right.$, where $R_{\mu \nu}$ is the Ricci tensor of the space) presents an obvious difficulty, due to the highly nonlinear nature of the equations. In spite of this fact, many solutions are known ${ }^{1}$; such solutions are usually degenerate in the sense that they correspond to space-times of an algebraically special type or to space-times which possess definite symmetries. Systems with two commuting Killing vectors have been thoroughly studied in recent years with the help of a variety of different solution-generating techniques. It is the aim of the present Letter to express the Einstein equations in such a form that techniques such as the inverse scattering method $^{2}$ or the use of Bäcklund transformations, ${ }^{3}$ which apply to the special case just mentioned, could conceivably be extended to the gen eral case.
The starting point in what follows will be the structural equations, as given for example by Ernst. ${ }^{4}$ Such equations admit an $\mathrm{SO}(3,1)$ - or $\mathrm{O}(3, C)$-invariant formulation; they will be written here as $\mathrm{SL}(2, C)$-invariant (spinor) equations. The introduction of spinors seems rather fundamental for the purpose of obtaining a very simple final form. Explicitly writing down dotted and undotted indices is not necessary in the present context, and as a consequence the equations may be expressed in a coordinate-free manner.

The basic quantities to be considered are the tetrad of one-forms $(k, m, t, \bar{t})$ and the connection one-forms $(u, v, w)$, where $u, v$, and $w$ are complex, while $k$ and $m$ are real and $\bar{t}$ is the complex conjugate of $t$. With a definite choice of the possible signs appearing in Ref. $4\left(\epsilon_{1}=\epsilon_{2}=\epsilon_{3}=1\right)$, the structural equations take the form ${ }^{4}$

$$
\begin{aligned}
& d k=\frac{1}{2}(u+\bar{u}) \wedge k+\bar{v} \wedge t+v \wedge \bar{t}, \\
& d m=-\frac{1}{2}(u+\bar{u}) \wedge m+w \wedge t+\bar{w} \wedge \bar{t}, \\
& d t=-\bar{w} \wedge k-v \wedge m+\frac{1}{2}(u-\bar{u}) \wedge t, \\
& d v-u \wedge v=C_{2} m \wedge \bar{t}+C_{1}(k \wedge m+t \wedge \bar{t})+C_{0} k \wedge t-\frac{1}{12} R k \wedge t+\frac{1}{2} S_{k k} m \wedge t+\frac{1}{2} S_{k t}(k \wedge m-t \wedge \bar{t})+\frac{1}{2} S_{t} k \wedge \bar{t}, \\
& d u-2 w \wedge v=-2 C_{1} m \wedge \bar{t}-2 C_{0}(k \wedge m+t \wedge \bar{t})-2 C_{-1} k \wedge t-\frac{1}{12} R(k \wedge m+t \wedge \bar{t})+\frac{1}{2} S_{k \bar{t}} m \wedge t \\
& +\frac{1}{2} S_{t} \bar{t}(k \wedge m-t \wedge \bar{t})-\frac{1}{2} S_{m t} k \wedge \bar{t}, \\
& d w-w \wedge u=C_{0} m \wedge \bar{t}+C_{-1}(k \wedge m+t \wedge \bar{t})+C_{-2} k \wedge t-\frac{1}{12} R m \wedge \bar{t}+\frac{1}{2} S_{\bar{t}} \bar{t} m \wedge t-\frac{1}{2} S_{m} \bar{t}(k \wedge m-t \wedge \bar{t}) \\
& +\frac{1}{2} S_{m m} k \wedge \bar{t}
\end{aligned}
$$

where $C_{i}, S_{i j}$, and $R$ correspond to the decomposition of the Riemann tensor into its Weyl, traceless Ricci, and scalar-curvature parts. The vacuum equations are obtained by setting $S_{i j}=R=0$ in Eqs. (2a)-(2c). The relevant equations reduce in such a case to Eqs. (1a)-(1c) and the following ones:

$$
\begin{aligned}
& d v-u \wedge v=C_{2} m \wedge \bar{t}+C_{1}(k \wedge m+t \wedge \bar{t})+C_{0} k \wedge t, \\
& d u-2 w \wedge v=-2 C_{1} m \wedge \bar{t}-2 C_{0}(k \wedge m+t \wedge \bar{t})-2 C_{-1} k \wedge t, \\
& d w-w \wedge u=C_{0} m \wedge \bar{t}+C_{-1}(k \wedge m+t \wedge \bar{t})+C_{-2} k \wedge t .
\end{aligned}
$$

Equations (1a)-(1c) and (3a)-(3c) may be readily written as equations for spinor-valued forms by de- 
fining

$$
\eta \equiv\left[\begin{array}{cc}
-k & t \\
\bar{t} & m
\end{array}\right], \quad \gamma \equiv\left[\begin{array}{cc}
\frac{1}{2} u & -v \\
w & -\frac{1}{2} u
\end{array}\right]
$$

Equations (1a)-(1c) and $(3 a)-(3 c)$ then take the following form:

$$
\begin{aligned}
& d \eta=\gamma \wedge \eta-\eta \wedge \gamma^{\dagger}, \\
& d \gamma-\gamma \wedge \gamma=W \wedge \eta,
\end{aligned}
$$

where the wedge product is understood as matrix multiplication, with the exterior product replacing the ordinary one between entries. The dagger denotes Hermitian conjugation, and

$$
W=\left[\begin{array}{ll}
-C_{0}(k+m)-C_{-1} t+C_{1} \bar{t} & -C_{1}(k+m)-C_{0} t+C_{2} \bar{t} \\
C_{-1}(k+m)+C_{-2} t-C_{0} \bar{t} & C_{0}(k+m)+C_{-1} t-C_{1} \bar{t}
\end{array}\right] .
$$

The following observation will be crucial in what follows: Equation (4b) with $W$ given by Eq. (5) is precisely equivalent to

$$
(d \gamma-\gamma \wedge \gamma) \wedge \eta=0
$$

The Einstein equations in vacuum thus reduce to the system

$$
\begin{aligned}
& d \eta=\gamma \wedge \eta-\eta \wedge \gamma^{\dagger}, \\
& (d \gamma-\gamma \wedge \gamma) \wedge \eta=0,
\end{aligned}
$$

with $\eta$ and $\gamma$ matrix one-forms satisfying $\eta^{\dagger}=\eta$, $\gamma \in \operatorname{sl}(2, C)(\operatorname{Tr} \gamma=0)$. A trivial computation shows that the system $(6 a)-(6 b)$ is closed under exterior differentiation, and as a consequence no further integrability conditions need to be considered. For the present purposes, this represents an advantage over the equations in the form (4a)-(4b), where a Bianchi-type identity should be explicitly taken into account.

The metric $g$ is given in terms of $\eta$ by means of the expression $g=\operatorname{Tr}(\eta \epsilon \otimes \bar{\eta} \epsilon)$, where $\epsilon=\left(\begin{array}{cc}0 & 1 \\ -1 & 0\end{array}\right)$. Equations (6a)-(6b) are manifestly invariant under the $\mathrm{SL}(2, C)$ gauge transformations

$$
\begin{aligned}
& \eta \rightarrow \eta^{\prime}=S \eta S^{\dagger}, \\
& \gamma-\gamma^{\prime}=S \gamma S^{-1}+d S S^{-1},
\end{aligned}
$$

where $S$ is an ordinary $\mathrm{SL}(2, C)$ matrix of arbitrary functions. Notice that Eqs. (6a) and (6b) may alternatively be written as the algebraically equivalent system

$$
d \eta=\gamma \wedge \eta-\eta \wedge \gamma^{\dagger}, \quad d(\gamma \wedge \eta)=\gamma \wedge \eta \wedge \gamma^{\dagger} .
$$

The vacuum equations (6a)-(6b) should be compared with the equations for flat space (vanishing Riemann tensor, $R_{\alpha \beta \mu \nu}=0$ )

$$
\begin{aligned}
& d \eta=\gamma \wedge \eta-\eta \wedge \gamma^{\dagger}, \\
& d \gamma-\gamma \wedge \gamma=0 .
\end{aligned}
$$

The system (8a)-(8b) may be obtained as the integrability conditions for the following system:

$$
\begin{aligned}
& \varphi \eta \varphi^{\dagger}=d \xi, \\
& d \varphi=-\varphi \gamma,
\end{aligned}
$$

where $\varphi$ and $\xi$ are matrix zero-forms with $\varphi$ $\in \mathrm{SL}(2, C)$ and $\xi^{\dagger}=\xi$. The general solution of Eqs. (8a) and (8b) may be extracted from (9a) and $(9 b)$ :

$$
\eta=\varphi^{-1} d \xi\left(\varphi^{\dagger}\right)^{-1}, \quad \gamma=-\varphi^{-1} d \varphi .
$$

This case is obviously "pure gauge," and may be reduced to the Minkowski form by means of a gauge transformation of the type (7a)-(7b), with $S=\varphi$, resulting in the standard expressions

$$
\eta_{0}=d \xi, \quad \gamma_{0}=0 \text {. }
$$

It is highly desirable to find a set of equations similar to (9a)-(9b), whose integrability conditions will consist of the field equations (6a)-(6b). This would permit the introduction of techniques similar to the inverse scattering method and Bäcklund transformations to the general case (Einstein equations with no isometries present). The equation

$$
(d \varphi+\varphi \gamma) \wedge \eta=0
$$

suggests itself as a candidate for generalizing Eq. (9b). Unfortunately, one is prevented from following this procedure by the fact that the equation $A \wedge \eta=0$, where $A$ is any matrix one-form, implies $A=0$, so that Eq. (10) would reduce to the trivial situation described by Eq. (9b). The problem is solved by using higher-order forms. Let $\Phi$ now be a matrix-valued one-form. The system

$$
\begin{aligned}
& \Phi \wedge \eta \wedge \Phi^{\dagger}=d \xi, \\
& d \Phi \wedge \eta=\Phi \wedge \gamma \wedge \eta
\end{aligned}
$$


(where $\xi$ is a matrix two-form with $\xi^{\dagger}=-\xi$ ) has Eqs. (6a)-(6b) as a sufficient condition for its integrability. Conversely, if we assume that there exists a sufficient number of independent $\Phi_{i}(i$ $=1, \ldots, N)$, the system of equations

$$
\begin{aligned}
& \Phi_{i} \wedge \eta \wedge \Phi_{j}{ }^{\dagger}=d \xi_{i j} \quad\left(\xi_{i j}{ }^{\dagger}=-\xi_{j i}\right), \\
& d \Phi_{i} \wedge \eta=\Phi_{i} \wedge \gamma \wedge \eta,
\end{aligned}
$$

implies the field equations as integrability conditions: By exterior differentiation of (12a) and (12b) one has

$$
\begin{aligned}
d \Phi_{i} \wedge \eta \wedge & \Phi_{j}-\Phi_{i} \wedge d \eta \wedge \Phi_{j}{ }^{\dagger} \\
+ & \Phi_{i} \wedge \eta \wedge d \Phi_{j}^{\dagger}=0, \\
d \Phi_{i} \wedge d \eta & =d \Phi_{i} \wedge \gamma \wedge \eta-\Phi_{i} \wedge d \gamma \wedge \eta \\
& +\Phi_{i} \wedge \gamma \wedge d \eta .
\end{aligned}
$$

Using (12b) and its Hermitian conjugate in Eq. (13a) one gets

$$
\Phi_{i} \wedge\left(\gamma \wedge \eta-d \eta-\eta \wedge \gamma^{\dagger}\right) \wedge \Phi_{j}{ }^{\dagger}=0 \text { 。 }
$$

For an appropriate number of independent matrices $\Phi_{i}$ this implies

$$
d \eta=\gamma \wedge \eta-\eta \wedge \gamma^{\dagger}
$$

With use of (14) and (13b), the following result is obtained:

$$
\Phi_{i} \wedge(d \gamma-\gamma \wedge \gamma) \wedge \eta=0 。
$$

Again, this implies

$$
(d \gamma-\gamma \wedge \gamma) \wedge \eta=0
$$

provided that enough $\Phi_{i}$ exist. The existence of a sufficient number of independent matrix oneforms $\Phi_{i}$ is equivalent to the existence of a solution of Eqs. (11a) and (11b) with a sufficient num- ber of parameters or functions. As an illustration of the use of Eqs. (11a)-(11b), it may be seen that the $p p$-wave metric ${ }^{1}$

$$
d s^{2}=2 d \zeta d \bar{\zeta}-2 d u d v-2 H d u^{2}
$$

[where $H=f(\zeta, u)+\bar{f}(\bar{\zeta}, u)$, with $f$ an arbitrary function of $\zeta$ and $u$ ] can be reconstructed from Eqs. (11a)-(11b) with a "scattering form" $\Phi$ given by

$$
\Phi=\left[\begin{array}{cc}
d \sigma-H_{\zeta} \psi d u & d \psi \\
d \lambda-H_{\zeta} \theta d u & d \theta
\end{array}\right]
$$

where $\sigma, \psi, \lambda$, and $\theta$ are arbitrary complex functions and $H_{\zeta} \equiv \partial H / \partial \zeta$. Whether a situation such as this is generic [namely, whether $\eta$ and $\gamma \wedge \eta$ may be obtained algebraically by solving equations such as (11a)-(11b) or (12a)-(12b) in the general case, with the $\Phi_{i}$ as an input] is not clear at present and requires further investigation. This matter is currently being considered.

The author wishes to thank Dr. F. Guil for numerous discussions.

Partial financial support by Comisión Asesora de Investigación Científica y Técnica is gratefully acknowledged.

${ }^{1}$ D. Kramer, H. Stephani, E. Herlt, and M. MacCallum, Exact Solutions of Einstein's Field Equations (Cambridge Univ. Press, Cambridge, 1980).

${ }^{2}$ V. A. Belinskiî and V. E. Zakharov, Zh. Eksp. Teor. Fiz. 75, 1955 (1978) [Sov. Phys. JETP 48, 985 (1978)].

${ }^{3}$ B. K. Harrison, Phys. Rev. Lett. 41, 1197, 1935 (E) (1978); G. Neugebauer, J. Phys. A 12 , L67 (1979); F. J. Chinea, Phys. Rev. Lett. 50, 221 (1983).

${ }^{4}$ F. J. Ernst, J. Math. Phys. 19, 489 (1978). 